
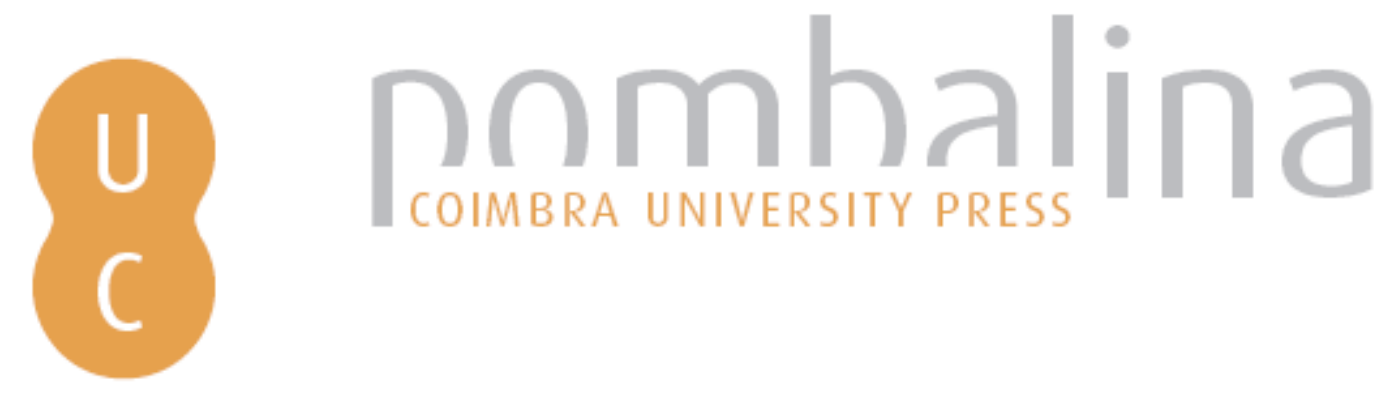

\title{
O impacto das fontes electrónicas de informação nas bibliotecas académicas portuguesas: uma avaliação quantitativa
}

\author{
Autor(es): $\quad$ Melo, Luiza Baptista; Pires, Cesaltina \\ Publicado por: Imprensa da Universidade de Coimbra \\ URL \\ persistente: URI:http://hdl.handle.net/10316.2/31900 \\ DOI: $\quad$ DOI:http://dx.doi.org/10.14195/978-989-26-0869-3_13 \\ Accessed : $\quad$ 26-Apr-2023 11:42:11
}

A navegação consulta e descarregamento dos títulos inseridos nas Bibliotecas Digitais UC Digitalis, UC Pombalina e UC Impactum, pressupõem a aceitação plena e sem reservas dos Termos e Condições de Uso destas Bibliotecas Digitais, disponíveis em https://digitalis.uc.pt/pt-pt/termos.

Conforme exposto nos referidos Termos e Condições de Uso, o descarregamento de títulos de acesso restrito requer uma licença válida de autorização devendo o utilizador aceder ao(s) documento(s) a partir de um endereço de IP da instituição detentora da supramencionada licença.

Ao utilizador é apenas permitido o descarregamento para uso pessoal, pelo que o emprego do(s) título(s) descarregado(s) para outro fim, designadamente comercial, carece de autorização do respetivo autor ou editor da obra.

Na medida em que todas as obras da UC Digitalis se encontram protegidas pelo Código do Direito de Autor e Direitos Conexos e demais legislação aplicável, toda a cópia, parcial ou total, deste documento, nos casos em que é legalmente admitida, deverá conter ou fazer-se acompanhar por este aviso.

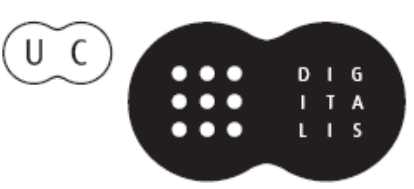


Maria Manuel Borges

Elias Sanz Casado

Coordenação

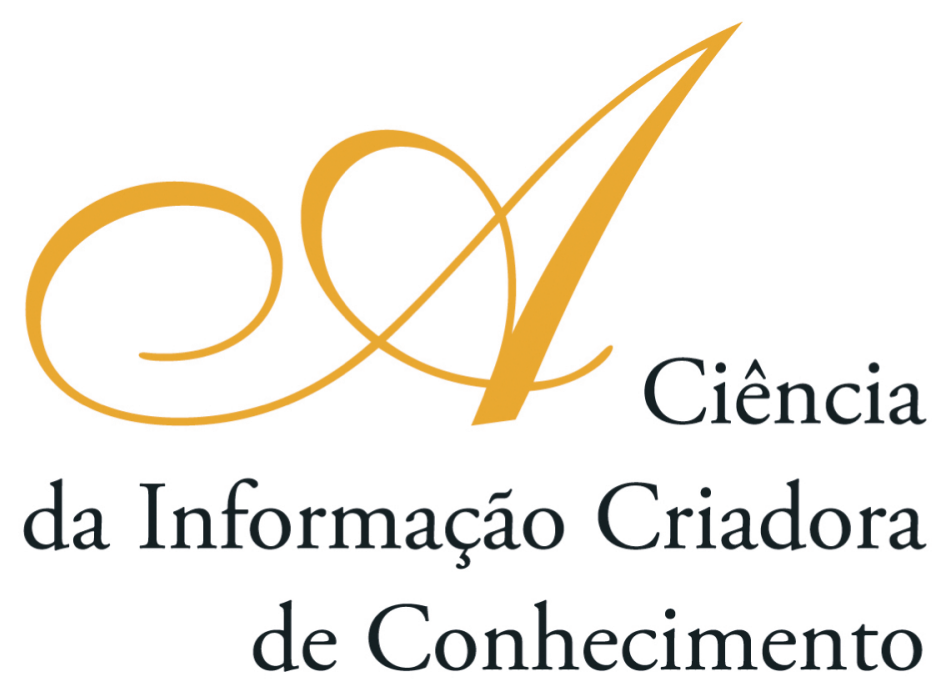

Vol. I I

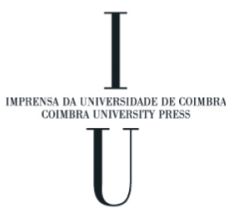

- COImbra 2009 


\title{
O Impacto das Fontes Electrónicas de Informação nas Bibliotecas Académicas Portuguesas: uma avaliaçấo quantitativa
}

\author{
Luiza Baptista Melo \\ Universidade de Évora e Universidade do Porto (Portugal) \\ Cesaltina Pires \\ Universidade de Évora (Portugal)
}

\section{Resumo}

Nas últimas duas décadas observaram-se grandes alterações nos serviços das bibliotecas académicas portuguesas. Essas transformações incluem a automatização dos catálogos, o acesso a bases de dados bibliográficos e de publicaçóes periódicas científicas, em texto integral.

$\mathrm{Na}$ actualidade, a biblioteca tradicional e a digital coexistem e completam-se nos serviços de documentação das Universidades e do Ensino Politécnico. A comunidade académica de Portugal tem acesso, via Internet, a grande quantidade de informação científica de qualidade, adquirida através de pagamento, e em Acesso Aberto.

O Estado português tem vindo a realizar grandes investimentos quer em infra-estruturas quer em informação. O principal objectivo é proporcionar acesso fácil e rápido a fontes de informação científicas. Esse processo pretende ser uma alavanca para promover o desenvolvimento do País. Neste contexto, é útil observar e estimar quais são os retornos destes investimentos nas bibliotecas e centros de documentação do Ensino Superior.

O presente estudo tem enfoque na identificação do impacto das fontes electrónicas de informação científica nas bibliotecas académicas portuguesas. Esta comunicação apresenta resumidamente um projecto de aferição que faz uma abordagem baseada nos seguintes objectivos:

- Estabelecer perfis de utilizadores destes serviços, por tipologia e áreas do conhecimento, baseados em aferiçóes de acordo com os indicadores de desempenho normalizados definidos pelas International Standards ISO 11620:1998, Amendment 1:2003 additional Information and documentation - performance indicators for libraries e ISO 2789:2006 Information and documentation - international library statistics.

- Observar dados bibliométricos e estabelecer relaçóes em função da utilizaçáo das fontes electrónicas de informação acessíveis na rede das instituiçôes públicas de Ensino Superior.

- Determinar o valor económico, em termos monetários, dos serviços electrónicos de informação científica fundamentado no Método de Avaliação Contingencial (MAC) e estimar a razão Custo/Benefício

\section{Abstract}

In the last 20 years there have been big changes in the Portuguese academic libraries services as academic libraries over the world. These changes include the adoption of automatic catalogues and the electronic access to bibliographic data bases and scientific journals in full text. Nowadays physical and digital sources are together. Professors, students and researchers are able to access simultaneous to a great amount of quality information pay and open access. The Portuguese Government has been investing to improve the access to 
the production of knowledge so as to develop the country. It is important to know the return on the investments in university libraries. The purpose of this paper is to identify the impact of the electronic sources in the Portuguese academic libraries. This paper describes an ongoing project to assess:

- The use of electronic scientific information and the correlation to the scientific production of the Portuguese academic community according to standard performance measures indicators and bibliometrics data.

- The economic value of these library services. In order to estimate in monetary terms the value of these services for end-users we use the contingent valuation method (CVM). This study reports the results of a quantitative e-survey of academic community.

\section{Introdução}

No decorrer das últimas duas décadas, a tecnologia desenvolveu e provocou enormes alteraçôes nas bibliotecas académicas Portuguesas. Na actualidade, à biblioteca tradicional veio adicionar-se a biblioteca digital, ambas coexistem e completam-se nos serviços de documentação académicos. Os bibliotecários e gestores de informação têm, que gerir simultaneamente recursos humanos e de informação com, os seguintes objectivos finais: proporcionar um acesso rápido à informação; satisfazer as expectativas do utilizador e reduzir os custos dos serviços.

Tendo enfoque nestas metas, vários profissionais em todo o mundo investigaram e definiram grande quantidade de metodologias para optimizar o desempenho e a qualidade dos serviços prestados nas bibliotecas. Neste contexto, é importante avaliar os serviços, uma vez que, existe a necessidade de conhecer se "we are providing the best possible services at right people and at the right price” (Davis, 2008).

Este trabalho pretende estudar alguns aspectos dos resultados e dos impactos dos serviços electrónicos nas bibliotecas académicas portuguesas. A pesquisa progrediu em três etapas:

1 - Selecção dos indicadores de desempenho e do cenário do Método de Avaliação Contingencial (MAC);

2 - Recolha e análise de dados; e,

3 - Identificação do valor económico dos serviços electrónicos de informação das bibliotecas académicas em termos monetários, avaliação da utilização das fontes electrónicas de informação (que tipo de informação científica, onde é pesquisada e qual a frequência da procura).

A selecção dos indicadores de desempenho a avaliar foi elaborada com base nas Normas Internacionais ISO 11620:1998, Amendment 1:2003 additional performance indicators for libraries and ISO 2789:2006. Para estimar em termos monetários o consórcio Biblioteca do Conhecimento Online (b-on), disponível nas bibliotecas do Ensino Superior Público em Portugal considerou-se o Método de Avaliação Contingencial (MAC). O presente estudo reporta os resultados quantitativos obtidos de inquérito por sondagem realizado à comunidade académica portuguesa. 


\section{Metodologia}

A avaliação dos serviços electrónicos de informação das bibliotecas do Ensino Superior Público, em Portugal, foram realizadas com base nas Normas Internacionais ISO 11620:1998, Amendment 1:2003 additional performance indicators for libraries e ISO 2789:2006. Consideraram-se os seguintes indicadores de desempenho: percentagem da população alvo que utiliza as bibliotecas tradicionais; percentagem da população alvo que utiliza as bibliotecas digitais; percentagem da população alvo que utiliza tanto as bibliotecas tradicionais como as digitais; local onde acede aos serviços; utilização dos serviços (bases de dados bibliográficos, colecçôes electrónicas, revistas científicas em texto integral pagas ou revistas em Acesso Aberto) e a média de tempo ganho pelos utilizadores destes serviços.

Oliveira e Coelho (2007) afirmam que "a metodologia de Avaliação Contingencial consiste em simular um mercado hipotético para o recurso em avaliação, contemplando diferentes níveis de provisão, e inquirir directamente os indivíduos sobre a sua disponibilidade em pagar por esse serviço (willingness to pay, WTP) e/ou disponibilidade em aceitar uma compensação pela potencial perda do mesmo (willingness to accept, WTA)". Nas últimas décadas vários autores recorreram à metodologia da Avaliação Contingencial para valorar em termos monetários os serviços das bibliotecas (Holt, G.E.; Elliott, D. \& Moore, A., 1999; McDermott Miller, 2002; Morris, A.; Sumsion, J. \& Hawkins, M., 2002; Holt, G.E. \& Elliott, D.S., 2003; British Library, 2004; Barron et al., 2005; Aabø, 2005; Morris; Ayre \& Jones, 2006; Elliott, D. S. et al., 2007; Hider, 2008) públicas, (Harless \& Frank, 1999) académicas, (Chung, 2007) e especializadas.

Nesta investigação implementou-se a metodologia de Avaliação Contingencial para estimar em termos monetários o consórcio Biblioteca do Conhecimento Online (bon). A metodologia desenvolvida baseia-se no tempo e nos custos recuperados, pelos utilizadores, e nos benefícios gerados pelo acesso às fontes electrónicas de informação científica disponíveis nas instituiçôes Públicas de Ensino Superior em Portugal.

\section{Recolha de dados}

A recolha de dados procedeu-se através de questionários. Inicialmente, efectuou-se um pré-teste com 50 questionários, em suporte papel. Após essa etapa, a recolha de dados realizou-se via questionário electrónico. Costa (2008, p.58) afirma que Jenkins indica as seguintes vantagens no emprego deste tipo de questionário: "acessível a toda a população da amostra, rápido feedback, recolha estandardizada da informação, possibilidade de monitorização dos resultados e redução de custos”.

O software livre Lime Survey, foi o programa implementado para a construção do questionário. Este software encontra-se disponível na Internet em http://www. limesurvey.org/ . Foram enviadas mensagens, via correio electrónico, à comunidade académica (trinta e duas instituiçóes de Ensino Superior Público e uma instituição de Ensino Superior Privado), solicitando a resposta a um questionário (que se encontrava no endereço indicado http://demos.neoscopio.com/neoasking/index. php?sid=51976\&lang=pt), de 15 de Janeiro a 15 de Março de 2009. Neste artigo, 
apresentam-se os resultados preliminares referentes ao período de tempo indicado. A recolha de dados deste projecto de avaliação ainda se encontra em curso.

O questionário baseou-se em indicadores de desempenho definidos nas Normas Internacionais ISO e no Método de Avaliação Contingencial (MAC), para avaliar os serviços electrónicos de informação das bibliotecas académicas portuguesas. $\mathrm{O}$ cenário hipotético criado para esta aferição, com base no MAC, fundamentou-se na seguinte ideia:

Suponha que o consórcio da b-on deixava de existir, não sendo possível aceder a estes serviços através da sua instituição. Contudo, continua a ser possível aceder aos serviços individualmente através do pagamento de uma mensalidade. Qual é o máximo que estaria disposto a pagar para continuar a aceder aos recursos da b-on, tendo em consideração os benefícios de tempo, esforço, custos de deslocação, impacto no número de artigos publicados, além de outros factores, numa escala de 5 Euros a 50 Euros? (Melo e Pires, 2009). A escala de valoração considerada no estudo baseouse em dados da FCCN (2008).

Existindo um enorme número de potenciais utilizadores destes serviços, o questionário foi realizado a uma amostra da população académica portuguesa. Neste estudo, ao inferirmos resultados utilizámos a amostra estratificação em cada instituição que participou no projecto de avaliação.

\section{Análise de dados}

A primeira fase deste projecto concretizou-se com a recolha de dados via questionário electrónico. A fase seguinte envolveu a comparação entre os diferentes tipos de utilizadores e as várias áreas do conhecimento. Foram considerados quatro grupos de utilizadores Professor/Investigador, Estudante de Doutoramento, Estudante de Licenciatura/Mestrado e Outro (incluí funcionários das bibliotecas académicas, administrativos e qualquer outra pessoa da comunidade académica). Assumiuse um conjunto de seis áreas disciplinares que agregam as seguintes Ciências do Conhecimento:

- Ciências Físico-Químicas (Física, Astronomia, Química e Ciências dos Materiais);

- Ciências Sociais e Humanas (Ciências Sociais, Economia, Contabilidade, Gestão, Finanças, Ciências Humanas e Bibliotecas e Ciências da Informação);

- Ciências da Terra e do Espaço (Ciências do Ambiente, Ciências da Terra e do Espaço);

- Ciências da Vida e da Saúde (Veterinária, Farmacologia, Enfermagem, Neurociências, Medicina, Imunologia e Microbiologia, Profissóes na área da Saúde, Estomatologia, Agricultura, Ciências Biológicas, Bioquímica, Genética e Biologia Molecular);

- Engenharia (Engenharia, Energia e Engenharia Química)

- Matemática e Ciências dos Computadores (Matemática e Ciências dos Computadores).

$\mathrm{Na}$ análise dos dados recorreu-se à estatística descritiva (tabelas de frequência e gráficos). Para vários pares de variáveis construíram-se tabelas de contingência e efectuaram-se testes de Qui-Quadrado (X2). Maroco (2007, p.103) explica que: 
O teste de Qui-Quadrado (X2) serve para testar se duas ou mais populaçôes (ou grupos) independentes diferem relativamente a uma determinada característica, i. e. se a frequência com que os elementos da amostra se repartem pelas classes de uma variável nominal categorizada é ou não idêntica.

Um teste de independência avalia se pares de observaçôes de duas variáveis, em tabelas de contingência, são independentes, uma da outra - por exemplo, se utilizadores de diferentes grupos apresentam diferentes frequências de utilização das revistas electrónicas científicas (Melo e Pires, 2009).

$\mathrm{Na}$ análise estatística efectuaram-se cálculos com os programas informáticos EXCEL e SPSS (Statistical Package for the Social Sciences).

\section{Descrição da Amostra}

Este projecto de avaliação baseou-se num questionário electrónico enviado à comunidade académica de todas as instituiçôes de Ensino Superior Público em Portugal (professores, investigadores, estudantes, funcionários das bibliotecas, administrativos e toda a gente que pudesse ser potencial utilizador dos recursos electrónicos científicos disponíveis nestas instituiçôes). Durante dois meses, receberam-se 1786 respostas completas. Dividiu-se essa população em quatro grupos: Professor/Investigador 32,4\%, Estudante de Doutoramento 7,8\%, Estudante de Mestrado/Licenciatura 27,8\% e Outro 32\%. Foram ainda consideradas seis áreas científicas: Ciências Físico-Químicas, Ciências Sociais e Humanas, Ciências da Terra e do Espaço, Ciências da Vida e da Saúde, Engenharia e Matemática e Ciências dos Computadores.

\section{Análise dos Resultados}

Nesta secção são apresentados os resultados preliminares do presente estudo. Iniciamos com uma análise dos dados obtidos nas respostas ao questionário electrónico. Estes dados são relevantes para conhecer os comportamentos dos utilizadores e as suas opiniôes sobre os serviços. Segue-se a análise dos resultados obtidos para os vários indicadores de desempenho e o cálculo dos valores Custo/Benefício, em função da média do tempo ganho por cada utilizador da Biblioteca do Conhecimento Online (b-on).

\section{Resultados para os indicadores de desempenho}

As Tabelas 1 e 2 sumarizam os dados obtidos nas 1786 respostas completas do questionário electrónico. $\mathrm{Na}$ Tabela 1 apresentam-se as percentagens de utilização dos vários tipos de bibliotecas e o local onde se acede aos recursos electrónicos de informação científica. $\mathrm{Na}$ Tabela 2 observam-se as percentagens dos utilizadores que conhecem a Biblioteca do Conhecimento Online (b-on) e as percentagens de utilizaçáo de várias ferramentas empregues para pesquisar a informação. 
Tabela 1 - Resultados das respostas ao questionário (percentagem da população alvo que utiliza as bibliotecas tradicionais, percentagem da população alvo que utiliza as bibliotecas digitais, percentagem da população alvo que utiliza tanto as bibliotecas tradicionais como as digitais e local onde acede aos serviços (Melo e Pires, 2009)

\begin{tabular}{|c|c|c|c|c|c|c|c|c|}
\hline \multirow{3}{*}{ Utilizadores } & Biblioteca & Biblioteca & Biblioteca & Não utiliza & \multicolumn{4}{|c|}{ Local onde acede à informação electrónica? (\%) } \\
\hline & Tradicional & Tradicional & Digital & Bibliotecas & \multirow{2}{*}{ Biblioteca } & Computador & $\begin{array}{c}\text { Campus } \\
\text { Universidade }\end{array}$ & \multirow{2}{*}{$\begin{array}{l}\text { Em } \\
\text { casa }\end{array}$} \\
\hline & $(\%)$ & Digital (\%) & $(\%)$ & $(\%)$ & & $\begin{array}{c}\mathrm{da} \\
\text { Universidade }\end{array}$ & no portátil & \\
\hline $\begin{array}{l}\text { Professor/ } \\
\text { Investigador }\end{array}$ & 6,7 & 63,3 & 27,2 & 2,8 & 6,7 & 55,4 & 54,0 & 51,4 \\
\hline $\begin{array}{l}\text { Est. } \\
\text { Doutoramento }\end{array}$ & 4,3 & 50,4 & 38,1 & 2,2 & 10,8 & 46,0 & 72,0 & 51,8 \\
\hline $\begin{array}{l}\text { Est.Mestrado/ } \\
\text { Licenciatura } \\
\end{array}$ & 45,7 & 40,4 & 4,4 & 9,5 & 42,5 & 27,6 & 58,9 & 47,7 \\
\hline Outro & 23,1 & 58,2 & 10,5 & 8,3 & 43,5 & 34,3 & 50,7 & 45,8 \\
\hline
\end{tabular}

Na Tabela 1 as percentagens presentes no primeiro item referem-se à utilização dos serviços, nos últimos 12 meses. Os valores variam entre 4,3 e 63,3 dependendo do tipo de serviço e dos vários utilizadores. Os valores superiores $(63,3 ; 50,4 ; 40,4$ e $58,2)$ indicam que a maioria dos utilizadores tem usufruído de ambas as bibliotecas, tradicional e digital, dos serviços académicos portugueses. O único grupo em que não se verifica essa situação é para o grupo Estudante Mestrado/Licenciatura que aparece com uma utilização superior nas bibliotecas tradicionais $(45,7)$. Pors (2006), numa investigação sobre os comportamentos informacionais de estudantes do ensino superior, concluiu que existem diferentes padróes de comportamento mas que estes baseiam-se nas exigências educativas. Se as necessidades educativas não incluírem requisitos que contemplem informação existente nas bibliotecas os estudantes evitam a utilização desses serviços. Este autor refere que os bibliotecários e profissionais da informação devem ter consciência deste facto, conhecer as necessidades de informação e o grau de interesse dos estudantes quando interagem com estes utilizadores.

É importante salientar que entre os grupos Professor/Investigador e Estudante de Doutoramento existe uma percentagem muito significativa que usufrui apenas da informação científica electrónica (27,2 e 38,1 respectivamente).

No item seguinte observam-se os locais onde os vários grupos acedem à informação electrónica (as percentagens variam de 6,7 a 72,0). Estes dados revelam que grande quantidade de utilizadores pesquisa informação no seu próprio portátil no campus da Universidade (por exemplo, 72,0\% dos estudantes de doutoramento realizam este procedimento. O grupo do Professor/Investigador acede à informação num computador da instituição mas fora das instalaçóes da biblioteca (55,4\%). Adicionalmente, cerca de $50 \%$ dos utilizadores acede a informação científica em sua casa via ligação à instituição onde exerce as suas actividades. Estes dados sugerem que muitos utilizadores acedam aos recursos electrónicos de informação científica durante a noite e no fimde-semana. 
Tabela 2 - Serviços utilizados por grupo de utilizador (Biblioteca do Conhecimento Online, bases de dados bibliográficos, revistas científicas em texto completo pagas e revistas em Acesso Aberto.

\begin{tabular}{|l|c|c|c|c|c|c|}
\hline \multirow{2}{*}{ Utilizador } & \multirow{2}{*}{ Conhece a } & \multicolumn{2}{|c|}{ Qual o tipo de recurso que utiliza? (\%) } & \multicolumn{2}{l|}{$\begin{array}{c}\text { Como procura a } \\
\text { informaçáo? (\%) }\end{array}$} \\
\cline { 2 - 7 } & \multirow{2}{*}{ b-on $(\%)$} & Bases & Revistas pagas & Informaçáo em & Catálogo de & \multirow{2}{*}{ Google } \\
\cline { 3 - 8 } & & bibliográficas & texto completo & Acesso Aberto & biblioteca & \\
\hline Professor/Investigador & 89,4 & 40,8 & 84,0 & 44,6 & 38,2 & 80,1 \\
\hline Est. Doutoramento & 87,1 & 41,0 & 79,9 & 52,5 & 36,0 & 82,0 \\
\hline Est.Mestrado/Licenciatura & 39,4 & 19,7 & 28,8 & 17,3 & 21,1 & 96,0 \\
\hline Outro & 62,8 & 30,9 & 50,2 & 35,3 & 43,4 & 89,9 \\
\hline
\end{tabular}

No primeiro item da Tabela 2 observam-se as percentagens de utilizadores que conhecem os serviços da b-on. Cerca de 90,0\% dos Professores, Investigadores e Estudantes de Doutoramento conhecem estas fontes de informaçáo electrónicas. Contrariamente, entre os Estudantes de Mestrado e de Licenciatura apenas 39,4\% admitem saber que existem estes recursos (foram calculadas separadamente as percentagens para estudantes de Licenciatura e para estudantes de Mestrado e verificaram-se resultados similares nos dois grupos). Estes dados sugerem que é urgente divulgar a existência destes serviços entre estes estudantes. Esta situação é particularmente relevante para os estudantes do $2^{\circ}$ ciclo universitário atendendo a que os programas a este nível requerem mais trabalho de investigação.

No segundo item, da Tabela 2, observa-se informação sobre as percentagens de utilização das bases de dados bibliográficos, revistas científicas pagas de texto integral e informação em Acesso Aberto. As revistas, adquiridas por compra, de texto completo apresentam as taxas mais elevadas de utilização de 84,0 para Professor/Investigador a 28,8 para Estudante Mestrado/Licenciatura.

$\mathrm{Na}$ Tabela 2, é incontestável que a maioria dos utilizadores recorre ao Google para encontrar informação na Internet (percentagens ente 80,1 e 96,0). O contrário parece ser válido em relação às frequências de utilização das bases de dados bibliográficas (valores entre 19,7 e 41,0) e aos catálogos das bibliotecas (valores entre 21,1 e 43,4). McMartin et al. (2008) referem que o Google (outros motores de pesquisa raramente mencionados) é o ponto de partida para muitas pesquisas na Internet. Na comunidade académica, muitos utilizadores reportaram que iniciam as suas pesquisas com o Google para descobrirem conteúdos que sabem que já existem, por exemplo, um website particular que faz ligação a livros em texto integral, associaçôes profissionais (académicas ou não académicas) ou instituiçôes governamentais. Este comportamento é um padrão comum de pesquisa (Smith, M. et al., 2003) e sugere que o Google tornou-se um instrumento seguro, identificado já em estudos anteriores (Manduca, C.A. et al., 2006; Pors, N.O, 2006).

McMartin et al. (2008) enfatizam que as ligaçôes entre as bibliotecas digitais educacionais e os motores de pesquisa criam um conjunto de sinergias propício ao acesso fácil a conteúdos de bibliotecas digitais e oferecem maior qualidade à informação acedida no WWW. 
Tabela 3 - Serviços utilizados por área do Conhecimento (bases de dados bibliográficos, revistas científicas em texto completo pagas e revistas em Acesso Aberto e tipo de pesquisa).

\begin{tabular}{|l|c|c|c|c|c|}
\hline \multirow{2}{*}{ Áreas do Conhecimento } & \multicolumn{2}{|l|}{ Tipo de recurso que utiliza (\%) } & \multicolumn{2}{l|}{$\begin{array}{l}\text { Como procura a } \\
\text { informaçáo (\%) }\end{array}$} \\
\cline { 2 - 6 } & Bases & Revistas pagas & Revistas & Google & Catálogo \\
\cline { 2 - 6 } & de dados & texto integral & Acesso Aberto & & Biblioteca \\
\hline Ciências Físico-Químicas & 41,1 & 76,1 & 29,4 & 90,6 & 28,9 \\
\hline Ciências Sociais e Humanas & 31,2 & 60,0 & 31,2 & 58,6 & 42,7 \\
\hline Ciências da Terra e do Espaço & 37,2 & 61,9 & 21,2 & 92,9 & 25,7 \\
\hline Ciências da Vida e da Saúde & 35,9 & 70,6 & 63,3 & 77,0 & 23,5 \\
\hline Engenharia & 21,7 & 36,5 & 16,3 & 95,4 & 34,4 \\
\hline $\begin{array}{l}\text { Matemática e Ciências dos } \\
\text { Computadores }\end{array}$ & 35,8 & 51,8 & 35,0 & 89,1 & 38,0 \\
\hline
\end{tabular}

O principal objecto desde projecto é analisar os impactos dos serviços electrónicos de informação científica na comunidade académica portuguesa e verificar os padróes de pesquisa e utilização destes recursos disponíveis nas instituiçóes de Ensino Superior. Na Tabela 3, exibem-se as frequências de utilização dos serviços por área de Conhecimento. Os dados revelam o seguinte:

- Os valores mais altos correspondem aos acessos a revistas pagas em texto integral;

- A comunidade académica acede a informação em Acesso Aberto, no entanto, são evidentes grandes variaçóes na utilização entre as várias áreas do Conhecimento. Realce para a percentagem de 63,3 do grupo de utilizadores das Ciências da Vida e da Saúde;

- Os catálogos das bibliotecas assim como as bases de dados bibliográficas são as fontes de menor utilização; e

- O grupo de utilizadores da área da Engenharia exibe as percentagens mais baixas de utilização dos vários serviços. No entanto, são os que mais utilizam o motor de pesquisa Google (95,4\%). Uma explicação possível encontra-se nas observaçóes, que já referimos anteriormente neste texto, sobre o padráo comum do comportamento na pesquisa de informação dos utilizadores.

\section{Análise Custo/Benefício}

A Tabela 4 apresenta a distribuição do tempo ganho pelos utilizadores quando acedem à Biblioteca do Conhecimento Online (b-on). Para cada grupo foi determinado o tempo médio ganho por mês. O tempo médio ganho para os grupos Professor/Investigador e Estudante de Doutoramento é razoavelmente elevado (8,79 e 9,85 horas por mês, respectivamente), porém para o grupo Estudante Mestrado/Licenciatura é de 4,4 horas por mês. A fase seguinte envolveu os cálculos da média do tempo ganho durante um ano e o tempo ganho por toda a comunidade académica portuguesa (professores, investigadores e estudantes), cerca de 402914 utilizadores, de acordo com dados do 
GPEARI (2008). O tempo adquirido pode ser convertido em termos monetários multiplicando o número de horas aproveitadas pelo valor do tempo de cada tipo de utilizador. Para estimar o valor do tempo ganho consideraram-se as tabelas salariais de cada grupo de utilizadores. Essa informação foi obtida da legislação portuguesa (valor dos salários dos professores e investigadores, bolsas de doutoramento, salários de monitores para os estudantes de Mestrado e Licenciatura). A percepçáo é que cada hora ganha com o acesso à informação científica, via Internet, pode ser utilizada noutra actividade (escrita de artigos científicos, investigaçáo no âmbito das teses de mestrado e doutoramento ou preparação de aulas); assim poder-se-á avaliar cada hora pelo seu valor de acordo com um aproveitamento alternativo. Estimando o valor do tempo ganho por todos os grupos de utilizadores obteve-se o benefício da Biblioteca do Conhecimento Online (b-on). De acordo com essa determinação calculou-se a razão Custo/Benefício 1:5,35.

Tabela 4 - Distribuição do tempo ganho pelos utilizadores do serviço b-on (Melo e Pires, 2009)

\begin{tabular}{|c|c|c|c|c|c|c|c|c|}
\hline \multirow{3}{*}{ Utilizador } & \multicolumn{7}{|c|}{$\begin{array}{l}\text { Quanto tempo ganha utilizando os serviços da b-on } \\
\text { (número de horas por mês)? }\end{array}$} & \multirow{2}{*}{$\begin{array}{l}\text { Tempo } \\
\text { médio } \\
\text { ganho }\end{array}$} \\
\hline & 0 & $1-3$ & $4-6$ & $7-9$ & $10-12$ & $13-15$ & 16 ou mais & \\
\hline & $(\%)$ & $(\%)$ & $(\%)$ & $(\%)$ & $(\%)$ & $(\%)$ & $(\%)$ & (horas/mês) \\
\hline Professor/Investigador & 6,7 & 14,0 & 20,4 & 11,6 & 10,0 & 5,9 & 20,8 & 8,79 \\
\hline Est. Doutoramento & 7,2 & 9,4 & 14,4 & 11,5 & 15,1 & 5,0 & 24,5 & 9,85 \\
\hline Est. Mestrado/Licenciatura & 5,2 & 14,5 & 11,1 & 5,2 & 2,2 & 0,6 & 1,0 & 4,44 \\
\hline Outro & 7,5 & 15,0 & 15,0 & 7,9 & 5,2 & 2,3 & 9,6 & 6,86 \\
\hline
\end{tabular}

A Tabela 5 apresenta a distribuição de frequências dos valores máximos que os utilizadores estáo dispostos a pagar (willingness to pay, WTP) para continuar a aceder aos serviços da Biblioteca do Conhecimento Online (b-on). O cenário hipotético considera que os custos mensais dos serviços da b-on, para cada utilizador, podem variar numa escala de 5 a 50 Euros.

$\mathrm{Na}$ Tabela 5, os resultados do lado esquerdo mostram que existe uma grande percentagem de utilizadores, que reclamam, isto é, não estão dispostos a pagar nada para continuar a usufruir dos serviços da b-on. Harless e Allen (1999) argumentam que, em estudos fundamentados no Método de Avaliação Contingencial (MAC), é comum existir um grupo de participantes que inicialmente recusa-se a responder qual o valor máximo WTP. Esta situação corresponde a um valor zero de protesto. Acreditamos que este problema também ocorreu no presente estudo. Para resolver esta situação, analisaram-se os dados de dois modos diferentes. Na primeira aproximação efectuaram-se os cálculos aceitando os valores nulos, isto é, os utilizadores que não estão dispostos a pagar nada pelos serviços. Esta aproximação sobrestima o verdadeiro valor WTP, porque apenas alguns zeros são respostas de protesto enquanto outros significam que o utilizador não dá uma valoração ao serviço b-on. Na segunda aproximação excluíram-se todos os utilizadores que declaram o valor WTP zero e considerou-se a distribuição da frequência entre os valores positivos. Esta aproximação subestima o valor total WTP, uma vez que, alguns zeros devem ser verdadeiros, isto 
é, alguns utilizadores podem não dar nenhum valor monetário aos serviços da b-on. Baseado nestas duas aproximaçóes é possível estimar um intervalo para o valor WTP dos utilizadores da Biblioteca do Conhecimento Online. Com base no Método de Avaliação Contingencial (MAC), calcularam-se as razóes Custo/Benefício aceitando os valores nulos 1:2,38 e excluindo os valores nulos 1:3,62.

Tabela 5 - Distribuição da frequências dos valores máximos que os utilizadores estão dispostos a pagar pelos serviços da Biblioteca do Conhecimento Online (b-on). O cenário hipotético considera que para se aceder à b-on é necessário pagar uma taxa mensal cujo valor pode varia numa escala de 5 a 50 Euros (Melo e Pires, 2009).

\begin{tabular}{|c|c|c|c|c|c|c|c|c|c|c|c|c|c|c|c|}
\hline \multirow{3}{*}{ Utilizador } & \multicolumn{15}{|c|}{$\begin{array}{l}\text { Qual é o máximo que estaria disposto a pagar para continuar a aceder aos e-recursos } \\
\text { (Euros por mêss)? }\end{array}$} \\
\hline & \multicolumn{8}{|c|}{ Aceitando os valores nulos } & \multicolumn{7}{|c|}{ Excluindo os valores nulos } \\
\hline & 0 & 5 & 10 & 15 & 20 & 30 & 40 & 50 & 5 & 10 & 15 & 20 & 30 & 40 & 50 \\
\hline Professor/Investigador & 27,5 & 24,0 & 20,0 & 6,9 & 10,1 & 4,6 & 0,8 & 6,1 & 33,1 & 27,6 & 9,5 & 13,9 & 6,4 & 1,1 & 8,4 \\
\hline Est. Doutoramento & 37,1 & 24,1 & 15,5 & 6,0 & 10,3 & 3,4 & 0,9 & 2,6 & 38,4 & 24,7 & 9,6 & 16,4 & 5,5 & 1,4 & 4,1 \\
\hline Est. Mes & 34,9 & 32,3 & 19,3 & 5,2 & 7,3 & 0,5 & 0,0 & 0,5 & 49,6 & 29,6 & 8,0 & 11,2 & 0,8 & 0,0 & 0,8 \\
\hline Outro & 36,2 & 28,9 & 17,2 & 4,7 & 7,0 & 3,2 & 0,4 & 2,9 & 45,2 & 26,9 & 7,3 & 11,0 & 5,0 & 0,0 & 4,6 \\
\hline
\end{tabular}

\section{Dados bibliométricos de Portugal}

O acesso às fontes electrónicas de informação científica nas bibliotecas académicas portuguesas, a nível nacional, teve o seu início em 2000. Esta iniciativa desenvolveu uma nova dinâmica no panorama do acesso, da comunidade académica e científica, à informaçáo de qualidade. Na Fig. 1 observam-se o número de artigos científicos portugueses publicados nas áreas de Ciências da Vida e da Saúde, Engenharia, Ciências Sociais e Humanas, Ciências da Terra e do Espaço, Ciências Físico-Químicas, Matemática e Ciências dos Computadores de 1996 a 2007.

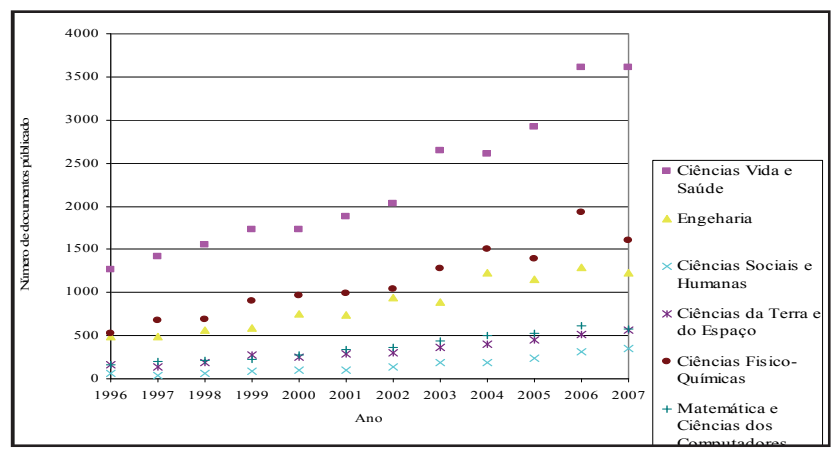

Fig. 1 - Número de documentos publicados nas áreas de Ciências da Vida e da Saúde, Engenharia, Ciências Sociais e Humanas, Ciências da Terra e do Espaço, Ciências Físico-Químicas, Matemática e Ciências dos Computadores de 1996 a 2007 (SCImago, 2007). 
É incontestável o aumento do número de documentos publicados desde 2001. Se compararmos o número de artigos publicados no período de 2005-2007 (os últimos três anos) com o número de artigos publicados no período de 1998-2000 (os últimos três anos antes do aparecimento das fontes electrónicas de informação, a nível nacional, nas instituições académicas públicas) concluímos que o número de documentos mais que duplicou (a taxa de crescimento é 105,6\%). Este aumento verifica-se em todos os domínios científicos com taxas de crescimento que variam de 92,2\%, em Engenharia, a 255,9\% em Ciências Sociais e Humanas (Melo e Pires, 2009).

\section{Conclusóes}

Lancaster (1993) afirmou que era excepcionalmente difícil, senão quase impossível, traduzir em termos monetários os benefícios dos serviços de uma biblioteca. No entanto, Hider (2008) refere que nas últimas décadas identificaram-se "correntes" de avaliação de bibliotecas com enfoque na quantificação do benefício do utilizador final. Este autor explica ainda que os estudos evoluíram no sentido de encontrar novos caminhos pelos quais a organização beneficie com os serviços de informação.

Adjacente a esta ideia, o estudo presente adianta algumas conclusóes preliminares de um projecto de avaliação das fontes electrónicas de informação disponíveis nas bibliotecas académicas portuguesas. Os resultados revelam que o comportamento da comunidade científica portuguesa em relação à procura e à utilização da informação electrónica científica tem vindo a mudar. $\mathrm{Na}$ actualidade, uma enorme percentagem de professores, investigadores e estudantes de doutoramento utilizam simultaneamente a biblioteca tradicional e a biblioteca digital. Quanto aos estudantes de mestrado e licenciatura apenas $40 \%$ utilizam ambos os serviços. Neste grupo de utilizadores $60 \%$ admite não conhecer os serviços da Biblioteca do Conhecimento Online (b-on). Este resultado não é surpreendente porque, neste grupo inclui-se o conjunto de estudantes do $1^{\circ}$ ciclo que não necessita de consultar informação tão especializada. Porém os resultados sugerem que é importante promover e informar sobre a existência destas fontes de informaçáo a estes potenciais utilizadores.

Os resultados realçam que a comunidade académica portuguesa utiliza os serviços electrónicos de informação científica durante a noite e ao longo do fim-de-semana.

Encontraram-se fortes evidências para afirmar que a comunidade académica indica que a primeira fonte de informação para acesso ao conhecimento é constituída pelas revistas científicas pagas em texto integral.

O Acesso Aberto à informação científica representa uma excelente fonte de informação disponível para a comunidade do Ensino Superior em Portugal.

Numa situação económica de crise mundial e fortes restrições orçamentais é particularmente interessante realizar estudos de Custo/Benefício das bibliotecas académicas para justificar às administraçóes das instituiçóes quais são os retornos dos investimentos. Neste artigo, tenta-se dar algumas respostas a estas questóes. Calculou-se a razão Custo/Benefício, para os serviços b-on, em função do tempo ganho obtendo-se a razão 1:5,35. Estimaram-se as razóes Custo/Benefício, fundamentadas no Método de Avaliação Contingencial (MAC), incluindo valores nulos, 1:2,38 e excluindo os valores nulos, 1:3,62. 
Perante estes resultados, conclui-se que os serviços da Biblioteca do Conhecimento Online (b-on), acessíveis nas bibliotecas académicas das instituiçóes públicas portuguesas, na globalidade, são valorizados acima do seu custo na perspectiva da população académica.

\section{Referências bibliográficas}

Aabø S. (2005). Are public libraries worth their price? A contingent valuation study of Norwegian public libraries. New Library World, 106(1218/129), 487-494.

Barron, D., Williams, R.V., Bajjaly, S., Arns, J. \& Wilson, S. (2005). The economic impact of public libraries on South Carolina. Columbia: University of South Carolina. Retrieved June 3, 2009, from http://www.libsci.sc.edu/SCEIS/home.htm

British Library (2004). Measuring Our Values. London: British Library. Retrieved January 15, 2007, from http://www.bl.uk/pdf/measuring.pdf

Chung, H.-K. (2007). Measuring the economic value of special libraries. The Bottom Line: Managing Library Finances, 20(1), 30-44.

Costa, M.T.F. (2008). O uso de periódicos científicos electrónicos nas instituiçōes do Ensino Superior Público em Portugal. Dissertação para a obtenção do Grau de Mestre em Ciências da Documentação e Informação - Faculdade de Letras da Universidade de Lisboa.

Davies, E. (2008). What does success look like? Evidence-based assessment of the academic library. In 17th Conference of Greek Academic Libraries: Academic library evaluation as a measure of Institutional Quality Assessment. Proceedings. Ioannina: University of Ioannina. Retrieved December 20, 2008, from http://17conf.lib.uoi.gr/files/a3.Davies.pdf

Elliott, D.S.; Holt, G.E., Hayden, S.W. and Holt, L.E. (2007). Measuring your library's value: how to do a cost-benefit analysis for public library. Chicago: ALA.

FCCN - Fundação para a Computação Científica Nacional (2008). B-on: Boletim Estatístico 2007. Lisboa: FCCN. Retrieved October 15, 2008, from http://www.b-on.pt/dmdocuments/ Boletim_estatistico_2007.pdf

GPEARI - Gabinete de Planeamento, Estratégia, Avaliação e Relaçôes Internacionais (2008) Estatísticas Nacionais: Ensino Superior. Lisboa: GPEARI. Retrieved July 28, 2008, from http://www.estatisticas.gpeari.mctes.pt/?action $=5 \&$ idt $=58$

Harless, D. W. \& Allen, F. R. (1999). Using the Contingent Valuation Method to Measure Patron Benefits of Reference Desk Service in an Academic Library. College \& Research Libraries, 60 (1), 56-69.

Hider, P. (2008). Using the contingent valuation method for dollar valuations of library services. Library Quarterly, 78(4), 137-158.

Holt, G.E.; Elliott, D. and Moore, A. (1999). Placing a value on public library services. Public Libraries, 38(2), 98-108.

Holt, G.E. and Elliott, D. (2003). Measuring outcomes: Applying cost-benefit analysis to middle-sized and smaller public libraries. Library Trends, 51(3), 424-440.

ISO 11620 (1998) Information and documentation - library performance indicators. Geneva: International Organisation for Standardisation.

ISO 11620 (2003). Information and documentation - library performance indicators: amendment1: additional performance des bibliothéques. Geneva: International Organisation for Standardisation. 
ISO 2789 (2006) Information and documentation - international library statistics. Geneva: International Organisation for Standardisation.

Kaufman, P. \& Watstein, S.B. (2008). Library value (return on investment, ROI) and the challenge of placing a value on public services. Reference Services Review, 36(3), 226-231.

Lancaster, F. W. (1993) If You Want to Evaluate Your Library (2nd ed.). Champaign: University of Illinois Press.

Manduca, C.A., Fox, S., et al. (2006). Digital library as network and community center. D-Lib, 12(12). Retrieved May 3, 2009, from http://www.dlib.org/dlib/december06/ manduca/12manduca.html

Marchioni, G. (2006). Exploratory search: from finding to understanding. Communications 49(4), 41-46.

Maroco, J. (2007). Análise estatística: com utilizaçâo do SPSS. Lisboa: Sílabo.

McDermott M. (2002) National Bibliographic Database and National Union Catalogue: Economic Evaluation for the National Library of New Zealand. Wellington: National Library of New Zealand. Retrieved December 14, 2006, from http://www.natlib.gov.nz/catalogues/librarydocuments/economic-valuation-nbd-nuc.pdf

McMartin, F., ·Iverson E.,.Wolf, A., Morrill, J., Morgan, G. \& Manduca, C. (2008). The use of online digital resources and educational digital libraries in higher education. International Journal of Digital Libraries, 9, 65-79.

Melo, L.B. \& Pires C. (2009). The impact of the electronic resources in Portuguese academic libraries. In Skiadas, C. H. (Ed.), QQML2009 - International Conference on Qualitative and Quantitative Methods in Libraries Proceedings. Chania: ASMDA International.

Missingham, R. (2005). Libraries and economic value: a review of recent studies. Performance Measurement and Metrics, 6(3), 142-158.

Morris, A., Ayres C. \& Jones A. (2006). Audiovisual materials in UK public libraries: economic sense?. Journal of Documentation, 62(5), 555-569.

Morris, A., Sumsion, J. \& Hawkins, M. (2002). Economic value of public libraries in the UK. Libri, 52, 78-87.

Oliveira, M.L. \& Coelho, M.P. (2007). Valorização de bens e serviços culturais: o método de avaliação contingencial aplicado ao caso das bibliotecas. In 9o Congresso Nacional de Bibliotecários, Arquivistas e Documentalistas, Ponta Delgada, Açores. Bibliotecas e arquivos: informação para a cidadania, o desenvolvimento e a inovação. Lisboa: B.A.D. Retrieved Maio 16, 2007, from http://badinfo.apbad.pt/Congresso9/COM9.pdf

Pors, N.O. (2006). Rationality and educational requirements: exploring students' information behaviour. In Ruthuen, I. (Ed.) Information Interaction Context, International Symposium on Information Interaction Context Proceedings from the Conference in Copenhagen 18-20 October, ACM International Press, pp. 288-300.

SCImago. (2007) SJR - SCImago Journal \& Country Rank. Retrieved April 24, 2009, from http://www.scimagojr.com

Smith, M., Barton, M., Bass, M., Branschofsky, M., McClellan, G., Stuve, D., Tansley, R. \& Walker, J.H.(2003). DSPACE: an open source dynamic digital repository. D-Lib Magazine 9(1). Retrieved May 14, 2009, from http://www.dlib.org/dlib/january03/smith/01smith. html 